Aus der II. chirurgischen Klinik in Wien (Vorstand: Prof. Hochenegg).

\title{
Zur chirurgischen Behandlung des Ascites.
}

Von Dr. Emil Schwarzmann, Operationszögling.

Bei dem allgemeinen Interesse, welches der operativen Behandlung des Ascites und ihren Resultaten zugewendet wird (man denke nur an die ausgedehnte Literatur über dieses Thema), erscheint es nützlich, über eine Reihe von i 4 Fällen zu berichten, die in den Jahren I go6--I I 12 wegen bestehenden Ascites an der II. chirurgischen Klinik operiert worden sind, zumal bei allen Kranken der weitere Verlauf des Leidens in Erfahrung gebracht werden konnte.

Bei oberflächlicher Betrachtung der großen Anzahl von Berichten über chirurgisch behandelte Ascitesfälle könnte man die Hinzufügung neuer Beobachtungen als überflüssig halten; wenn man aber bedenkt, wie individuell verschieden gerade die mit Ascites verbundenen Krankheitszustände verlaufen und wie rasch die chirurgischen Operationsmethoden für die Behandlung des Ascites einem Wandel unterlegen sind, dann wird man anderer Mcinung. Dazu kommen noch Bedenken, welche dem Leser der vorhandenen Statistiken aufsteigen und die bereits von Koch (I) am II. Kongre $\beta$ der internationalen chirurgischen Gesellschaft in Brüssel geäußert wurden, welche dic weitere Mitteilung von Beobachtungen wünschenswert erscheinen lassen. Koch beklagt sich über die Unzuverlässigkeit der Sammelstatistiken und meint, daß wir auf die Resultate derselben nicht vertrauen können.

In klarer Erkenntnis dieser Tatsache sagt La d en berger (2): „Wichtiger als Statistiken, die Fälle der verschiedensten Dignität zusammenfassen und schwer kontrolliert werden können, sind Einzelmitteilungen geheilter Fälle, die jahrelang verfolgt wurden."

Zur besseren Übersicht der operativen Erfolge führe ich aber 
in Kürze einige Daten aus den größeren bisher erschienenen Sammelstatistiken an:

\begin{tabular}{|c|c|c|c|c|c|c|}
\hline $\begin{array}{l}\text { Statistik } \\
\text { von }\end{array}$ & 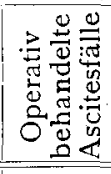 & 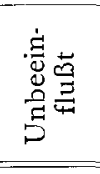 & 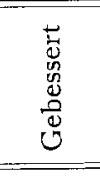 & $\begin{array}{l}\stackrel{5}{0} \\
\frac{0}{9} \\
0\end{array}$ & 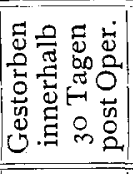 & Bemerkungen \\
\hline $\begin{array}{l}\text { Monprofit } \\
\text { I904 }\end{array}$ & 224 & $15 \%$ & $13 \%$ & $35 \%$ & $20 \%$ & - \\
\hline $\begin{array}{c}\text { Bunge } 4 \\
\text { I905 }\end{array}$ & 274 & $56 \%$ & $14 \%$ & $30 \%$ & $3,6 \%$ & - \\
\hline $\begin{array}{l}\text { Sinclair }^{5} \\
\text { White } \\
\text { I907 }\end{array}$ & 227 & $15 \%$ & I $3 \%$ & $37,3 \%$ & $33 \%$ & $\begin{array}{l}\text { Nur Leberzir- } \\
\text { rhosen }\end{array}$ \\
\hline $\begin{array}{l}\text { Oettinger } \\
1907\end{array}$ & 169 & $28,4 \%$ & $20,7 \%$ & $50,9 \%$ & - & - \\
\hline $\begin{array}{l}\text { Bircher } \\
\text { I909 }\end{array}$ & 343 & $48 \%$ & I $3 \%$ & $33 \%$ & - & - \\
\hline $\begin{array}{l}\text { Greenough } 8 \\
\text { I9I } 2\end{array}$ & 105 & $27,6 \%$ & $42 \%$ & - & $29,5 \%$ & $\begin{array}{l}\text { Reine unkompl. } \\
\text { Zirrhosen }\end{array}$ \\
\hline
\end{tabular}

Unter Heilung müssen wir hier natürlicherweise nur die Heilung eines Symptoms, des Ascites, verstehen; auf den Verlauf der Grundkrankheit haben die vorliegenden Zahlen keinen Bezug. Nehmen wir die geringste Heilungsziffer von 30 Proz. (Bunge) an, dann müßte wohl zugegeben werden, daß wir vor einem wirklichen, ganz unzweifelhaften Erfolg stchen, welchen uns der chirurgische Eingriff beschieden hat. Es sind ja einzelne Fälle von spontanem dauernden Rückgang des Ascites bei Leberzirrhose in der Literatur verzeichnet; auch fehlen uns nicht Angaben über Heilungen des Ascites nach bloßer Paracentese. Doch sind diese glücklichen Zufälle selten und erklären sich durch die Entwicklung von Kollateralen, die sich entweder spontan bilden oder von Verwachsungen, die von den Einstichen des Troikarts bei den zahlreichen Punktionen provoziert wurden. So berichtet Monprofit von einem Kranken, den er 20 mal punktierte und wo dann der Ascites 2 Jahre ausblieb, Macdonald (9) sah bei einem Kranken den Ascites nach der 6o. Punktion ausbleiben, während einer Beobachtungszeit von 4 Jahren (Med. News Okt. I889), Hawkins (IO) berichtet Ähnliches (Med. Presse 1908). Lantier (I I) teilt einen Fall 
mit (Journal de méd. de Bordeaux I908, Nr. 40), wo die Talmasche Opcration spontan realisiert war. Wir wissen, daßin in einer beträchtlichen Anzahl von Fällen sich das Netz an der vorderen Bauchwand bereits fixiert vorfindet. Immerhin handelt es sich um Zufälle, auf die man nicht rechnen darf. Gewöhnlich bedeutet das Auftreten des Ascites ein prognostisch sehr ernstes Symptom und die Frage, wie ihn der Arzt zu behandeln hat, ist eine höchst wichtige. Um uns mit der Beantwortung dieser Frage zu beschäftigen, wollen wir die Krankheitszustände Revue passieren lassen, welche mit Ascites einhergehen und die für eine chirurgische Behandlung in Betracht kommen. Kürzlich hat Cabot (American Journ. of the med. sciences I9I2) eine Studie über die Ursachen des $\Lambda$ scites angestellt. Die Schwierigkeiten in der Differentialdiagnose der dem Ascites zugrunde liegenden Erkrankungen veranlaßten ihn, an einem Material von 5000 Fällen die Häufigkeit der einzelnen Ursachen festzustellen. Als Ursache in 224 obduzierten und bei 3086 klinisch beobachteten Fällen fanden sich in

89 Fällen Herzschwäche,

28 , Neoplasmen des Peritoncums,

I6" Neoplasmen der Bauchorgane,

$26 ”$ Nephritis,

23 " Leberzirrhose,

Io " tuberkulöse Peritonitis,

5 " Tabes mesaraïca,

von 224 obduzierten Fällen,

der Rest fiel auf eine Anzahl anderer Befunde. In

1397 Fällen Herzschwäche,

703 „Herzklappenfehler (davon 67 Mitral- und Tricuspidalinsuff.),

665 Nierenaffektionen,

325 " Leberzirrhose (von 367 beobachteten Fällen),

263 " tuberkulöse Peritonitis,

36 " Herzbeutelverwachsung (von 47 beobachteten Fällen), von 3086 klinisch beobachteten Fällen.

Ziehen wir die von Ladenberger systematisch eingeteilten Indikationen für ein chirurgisches Eingreifen bei Ascites in Betracht, dann ist eine Operation indiziert bei 
I. Stenose des Pfortaderstammes,

2. Erkrankungen der Verzweigung der Pfortader in der Leber. Dazu gehören

a) die Leberzirrhose, und zwar sowohl die atrophische, als auch die hypertrophische Form mit Ikterus und Mischformen,

b) die Cirrhose cardiaque,

c) die Zuckergußleber und die Picksche perikarditische Pseudoleberzirrhose,

d) die Bantische Krankheit.

Wie wir aus den Cabotschen Untersuchungen ersehen, kommen von den vorstehenden Erkrankungen als verhältnismäßig häufig in Betracht vor allem die Leberzirrhosen, in zweiter Linie die Herzbeutelverwachsung (deren ätiologischer Zusammenhang mit den sub c) angeführten Erkrankungen bekannt ist). Als weitere Indikationen möchten wir die tuberkulöse Peritonitis und die Tricuspidalinsuffizienz hinzufügen.

Haben wir erwähnt, welche Erkrankungen als die häufigsten für die Behandlung des mit ihnen einhergehenden Ascites die größte Aufmerksamkeit verdienen, so wollen wir damit nicht von vornherein erklären, daß der Ascites chirurgisch behandelt werden müsse. Das ist eine Frage, deren Berechtigung noch zu erweisen ist. $\mathrm{Zu}$ diesem Behufe ist die Betrachtung der atrophischen Leberzirrhose und des sie begleitenden Ascites geeignet.

Der Verlauf einer atrophischen Leberzirrhose wird von den verschiedenen Autoren sehr verschieden angegeben. In $\mathrm{Ziems-}$ sens Handbuch sagt Thierfelder (I3): „Die Dauer des Krankheitsprozesses ist bei der Verborgenheit seines Anfangs nicht genau zu bestimmen. Die Zeit, welche vom Auftreten der ersten Krankheitserscheinungen bis zum Tode vergeht, schwankt in den verschiedenen Fällen zwischen sehr weiten Grenzen. Rechnet man von den Symptomen der Leberhyperämie an, die manchmal den Beginn der Wucherung des interstitiellen Gewebes zu begleiten scheint, so gibt es Fälle, in denen die Krankheit 3 Jahre und darüber dauert .... Der Ausgang ist in den sicher diagnostizierbaren Fällen ohne Ausnahme der Tod. Dr. Bristowe (14) (zit. nach W. Cheadle, Brit. med. Journ. I 892) sagt: „Der Verlauf 
der Zirrhose mag wohl einige Jahre dauern; aber wenn sich ausgesprochene Symptome der Erkrankung zeigen, dann sind die Tage des Patienten gezählt. Er dürfte dann kaum noch ein bis zwei Jahre weiterleben."

Cheadle sagte über die Prognose bei der atrophischen Zirrhose: „In jedem Falle, wo die Leber zusammengeschrumpft und klein war, trat bestenfalls eine kurzdauernde Erholung ein; hingegen äußert er betreffend die hypertrophische Zirrhose, „daß es keinen noch so hohen Grad von Abmagerung und von Wassersucht geben kann, der hier nicht die Möglichkeit einer Heilung hätte." Wiewohl sich im Laufe der Zeiten die Anschauungen über den Verlauf der Leberzirrhose in günstigem Sinne geändert haben, insbesondere infolge des Umstandes, daß man die anfänglichen Störungen zu Beginn der chronischen Leberentzündung mit in die Krankheitsdauer einrechnete (unter dieser Einschränkung konnte Hoppe-Seyler eine Dauer von io und mehr Jahren vermutungsweise schätzen), so gilt auch heute das voll entwickelte Krankheitsbild der Leberzirrhose in seiner Prognose als letal. „Hat man die Diagnose gestellt,“ sagt Pel (r6), „,so dauert das Leiden in der Regel höchstens ein paar Jahre."

Der Ascites stellt eines der unheilvollsten Symptome der Erkrankung dar. Ewald (I7) (Lebcrkrankheiten, Thieme, Leipzig 1913) beobachtete ihn in 63 Proz. sciner Fälle, White meint, $\mathrm{daB}$ er in 50 Proz. der Fälle vorkomme. Nach den vorhin erwähnten Untersuchungen von $C$ abot müßte man ihn viel häufiger erwarten. Jedenfalls sind die Störungen, die er hervorruft, wenn er die Peritonealhöhle erfüllt, deletäre: Es tritt Zwerchfellhochstand auf, die Atmung und die Herztätigkeit sind stark behindert, wodurch die Stauungshyperämie im Pfortadergebiet erhöht und stärkere Ascitesbildung bewirkt wird. Letztere führt bekanntlich auch häufig zu Pleuraerguß. So ergibt sich durch eine Art Circulus vitiosus ein in steter Progression anwachsender Erguß im Bauchraume, der den Kranken in einen lebendigen Wasserschlauch umwandelt. In diesem Zustande kann der Patient selbstverständlich nicht lange leben - eine Behebung des Ergusses tut dringend not; alle internen Mittel sind erschöpft, die Punktionen werden in immer kleineren Zwischenräumen nötig, der Säfteverlust bringt den Kranken um seine letzten Kräfte. 
Wenn also auch der klinische Verlauf der Leberzirrhose kein sehr ermutigender ist, so lohnt es sich doch, den Ascites, seine symptomatisch unangenehmste Komplikation auf das energischste zu bekämpfen.

Talma (18) einerseits, Drummond und Morison (19) andererseits haben wir das erste Verfahren zu verdanken, mittels dessen es gelungen ist, dieses quälendste Symptom zum Verschwinden $z u$ bringen. Sie nähten das Netz an die vordere Bauchwand an, bzw. auch die Milz, Leber und Gallenblase. Dadurch erzielten sie Zustände, wie sie die Natur bei der Spontanheilung des Ascites selbst hervorruft: sie eröffneten die Seitenbahnen für den Abfluß des gestauten Pfortaderblutes. Seit dem Jahre 1896 wird nun diese Talma-Drummond-Morisonsche Operation häufig geübt in ihren zahlreichen Modifikationen und Variationen. - Wir wollen jetzt eine kurze Übersicht über die seither ersonnenen Operationen zur dauernden Behebung des Ascites bei Stauungszuständen im Pfortadergebiete mitteilen, dann werden wir im Anschluß an die Anführung unserer Fälle die Operationsresultate besprechen und es wird schließlich versucht werden, die Frage zu beantworten, ob wir nicht durch die neuen Funktionsprüfungsmethoden der Leber zu einer Zeit kollaterale Blutbahnen eröffnen könnten, bevor sich die unheilvollen Folgen der Pfortaderstauung bemerkbar machen.

Is kann nicht meine Absicht scin, alle Eingriffe und Operationsmethoden zu schildern, welche ersonnen wurden, um das Symptom des Ascites zum Verschwinden zu bringen. Sie sind Legion. Ich will nur eine kurze Übersicht und Anführung der erwähnenswerten geben. Ihre Einteilung in 4 Gruppen ergibt sich von selbst:

I. Die Operation nach Talma-I)rummond und Morison und ihre Modifikationen.

2. Die Ruottesche (20) Operation („Saphenopexie“).

3. Die Verfahren, welche eine subkutane Drainage zum Ziele haben.

4. Die Anlegung einer Anastomose nach v. Eck und andere direkte Venenanastomosen.

Über die erste Gruppe von Operationen will ich mich ganz kurz äußern, denn sie wurden oft genug beschrieben. Talma 
nähte das Netz an das Peritoneum der vorderen Bauchwand an und fügte diesem Eingriff auch noch die Fixation der Milz (Narath) und Leber an das gegenüberliegende Peritoneum parietale hinzu. Drummond und Morison verbanden diese Operation mit einer suprapubischen Drainage (Brit. med. Journ. 1896). Schiassi implantierte das Netz präperitoneal zwischen die Schichten der vorderen Bauchwand. Sinclair White präparierte das Peritoneum parietale mit der hinteren Rektusscheide $\mathrm{ab}$ und bildete so einen Lappen, den er drehte und mit einigen Nähten in dieser gedrehten Lage fixierte. An diesen Lappen nähte er das Netz an. Außerdem rieb er die Unterfläche des Zwerchfells an die obere Fläche der Leber mit steriler Gaze kräftig ab und befestigte den vorderen Leberrand mit drei Nähten an das Zwerchfell, von denen eine den Peritonealüberzug der Gallenblase mitfaßte.

Erwähnt sei weiter der Vorschlag Omis und Itos (2I), die durch eine Tamponade der Bauchhöhle innige Verwachsungen der Darmschlingen untereinander und dieser mit der Bauchwand herbeiführen wollen, dann die von $\mathrm{O} \mathrm{mi}$ (22) durchgeführte intra. peritoneale Verlagerung der Niere oder die Einnähung des Netzes in einen Nephrotomieschnitt. Delagrenière (23) modifizierte die Talmasche Operation so, daß er Leber und Gallenblase an die vordere Bauchwand fixierte, das Netz jedoch über die Leber emporschlug und am Zwerchfell befestigte.

Dic „Saphenopexie“, eine der geistvollsten Operationen, entstammt einem französischen Chirurgen, $R$ u ot te. Bei einem alten Zirrhotiker führte er diesen Eingriff zum ersten Male am 29. I. 1907 aus. Er besteht darin, daB beide Venae saphenae jederseits in einer Entfernung von $8 \mathrm{~cm}$ vor ihrer Einmündung nach Ligierung des distalen Abschnittes durchschnitten und die proximalen Anteile in je eine etwa $2 \mathrm{~cm}$ große Öffnung des Peritoneum parietale, nach seitlicher Schlitzung ihrer Lumina, angenäht werden. Der direkte Abfluß der Ascitesflüssigkeit in die Blutbahn war dadurch ermöglicht und es ging bei diesem ersten Patienten der Ascites für immer zurück.

A. J. Morosowa (24) fand in der Literatur 15 Fälle dieser Opcration, von denen fast die Hälfte von Erfolg begleitet war (Ref. Zentralbl. f. Chir. I9I3). 
Der dritten Gruppe gehört die große Anzahl der Verfahren an, die den peritonealen Erguß in das subkutane Zellgewebe abzuleiten trachten. Bis zu einem gewissen Grade dürfte dieses Bestreben auch bei der subkutanen Verlagerung des Netzes nach Narath (25) (Zentralbl. f. Chir. 1905) zum Ausdruck kommen. In ausgesprochener Weise erstreben dieses Zicl eine ganze Reihe von Verfahren, von denen die einen

a) ohne Drains, die anderen

b) mit Drains oder drainähnlich wirkenden Vorrichtungen zur Anwendung kommen.

$\mathrm{Zu}$ den ersteren gehört die subkutane Fixation einer trichterförmigen Vorzichung des Bauchfells bzw. die Bildung einer Drainagelücke durch Benutzung einer von Peritoneum umsäumten Rektusdiastase (Handley, Evler [26]), zu den letzteren die Drainage mittels durchgezogener Seidenfäden als Dochte aus. der Bauchhöhle in die Subcutis (Lambotte [27]), die Herausleitung durch Glasdrains (Tavel [28]), Gummidrains (Paterson [29]), Silberdrahtbügel (Franke [30]), Gummi- oder Fischblasenkondoms (Henschen [3I $]$ ) und Silberröhrchen (Perimoff |32|). Schließlich erwähne ich die von Franke (33) (Rostock) am Hunde und zum ersten Male von Vidal (34) am Menschen mit Glück ausgeführte Vereinigung der Vena cava inf. mit der Pfortader (Ecksche Fistel); dieser Methode anzugliedern wäre noch der Versuch von Villard und Tavernier (35), die zur Behebung der Pfortaderstauung eine Anastomose zwischen einer Vena ovarica und der Vena mesenterica inferior anlegten.

Die I4 an unserer Klinik operierten Fälle möchte ich, entsprechend der in $\Lambda$ nwendung gebrachten Operationsmethoden in 6 Gruppen besprechen, wodurch sie sich übersichtlich verteilen.

I. Gruppe: Einfache Omentopexie.

Fall $\mathrm{I}$ u. 2.

II. Gruppe: Omentofixation in eine präperitoneale Tasche. Fall $3-6$.

III. Gruppe: Omentofixation in eine subkutane Tasche (nach Narath).

Fall 7 u. 8. 
IV. Gruppe: Präperitoneale Fixation der Milz plus Omentopexie.

Fall 9--I2.

V. Gruppe: Fixation des linken Leberlappens plus Omentopexie.

Fall I3.

VI. Gruppe: Omentopexie plus beiderseitige Saphenopexie. Fall I 4 .

I. Gruppe: Bei diesen Fällen wurde also das Netz an das Peritoneun parietale der vorderen Bauchwand fixiert. (Fall I und 2.)

Der erste Fall starb 47 Tage nach dem Eingriff zu Hause. Eine Obduktion wurde nicht vorgenommen und über die Art seines Todes war nichts Näheres zu eruieren.

Krankengeschichte:

A. B., 48 jähr. Mechaniker. Vor 3 Jahren Anschwellen der Füße am Abend (1906). Im November 1908 Anschwellen des Bauches, seit kurzer Zeit Atembeschwerden. Potus täglich 8-ro Viertel Wein. 20--25 Zigaretten täglich. Spitalaufenthalt: 27. II. bis 24. V. I909.

Aus dem Status praesens: Tremor beider Hände, Teint fahlgelb, Konjunktiven leicht ikterisch, Ödeme an den unteren Extremitäten. Herztöne rein. Über der linken Iungenspitze Schallverkürzung. Diffuse Bronchitis. In den unteren Lungenpartien Kompressionsatmen. Abdomen stark aufgetrieben, Decken straff gespannt, zahlreiche erweiterte Hautvenen über dem Abdomen. Nabel verstrichen. Ascites. Harn: spez. Gew.: 1027, kcin Albumen, Gallenfarbstoffe: Spuren, Indikan nicht vermehrt, Urobilin + , Urobilinogen +- , Wassermann negativ, Bauchumfang $110 \mathrm{~cm}$.

I. III. Igog Punktion: Entleerung von $7 \frac{1}{2}$ Liter klarer seröscr Flüssigkeit von sp. G. IoIo. Nach der Punktion Hautvenen nicht mehr deutlich sichtbar, Leber hart, von höckriger Oberfläche, Rand scharf, keine Druckempfindlichkeit. 3 Stunden.

2. III. $20 \mathrm{~g}$ Galaktose per os; Ausscheidung von $\mathrm{I}, 2 \mathrm{~g}$ in

Der Patient wird mit der Diagnose bakteriotoxische Zirrhose an unsere Klinik transferiert (I5. IV. 1909).

Am 17. IV. Omentopexie. Dic Leber zeigt sich derb, zirrhotisch, vergrößert.

24. IV. Heilung der Wunde p. pr. Der Patient wird am I. V. 1909 in gebessertem Zustande entlassen, stirbt aber nach 47 Tagen in seiner Wohnung. 
Der zweite Fall (Nr. 2) betraf eine Tuberkulose des Bauchfells. Die Leber bot intra operationem das Bild einer großhöckerigen Zirrhose. Die Patientin überlebte den Eingriff I 3 Monate; wiewohl sich post operationem ein Caput Medusae entwickelte, war in der Folgezeit alle 2 Wochen eine Punktion nötig. Es kann hier demnach von keinem rechten Erfolg gesprochen werden.

\section{Krankengeschichte:}

M. K., 20 jähr. Private aus Rußland (Spitalaufenthalt vom 24. X. I9IO bis 20. III. I9II). War bis vor 3 Jahren gesund; erkrankt um diese Zeit an Schmerzen in der Ileocöcalgegend, die mit Diarrhöen und häufigem Erbrechen einhergehen. Kurzdauernde Besserung, die wiederum durch Beschwerden unterbrochen wird. Blutige Stühle, häufig Diarrhöen. Zunehmen des Bauchumfangs; Atembeschwerden. Seit 8 Wochen Husten und Auswurf. Gewichts. abnahme.

Aus dem Status praesens (25. X. I910). Blasses, zart gebautes Mädchen. Herzbefund normal. Dämpfung über beiden Lungenspitzen. Bauchumfang $83,5 \mathrm{~cm}$. Starke Diastose der Musculi recti. Milztumor. Ascites. Ödeme der unteren Extremitäten. Im Harn Albumen. Wassermann negativ. Calmette deutlich positiv. Auf $40 \mathrm{~g}$ Galaktose per os werden in den nächsten 4 Stunden $2,8 \mathrm{~g}$ ausgeschieden.

27. X. Punktion des Ascites: Es werden 5 Liter abgelassen: trübe, sanguinolente Flüssigkeit vom sp. G. roo8, im Zentrifugate vorwiegend Lymphocyten enthaltend und grampositive Diplokokken.

Die Pat. wird mit der Diagnose: tuberkulöse Peritonitis von der internen Klinik zu uns transferiert und am 20. II. operiert. Die Leber ist stark geschrumpft und bietet das Bild einer großhöckerigen Zirrhose. Die Wundheilung erfolgte p.pr. Hier wurde am 2o. IV. I9I I 2 Monate post operationem der Galaktoseversuch wiederholt. Diesmal wurden von $40 \mathrm{~g}$ Galaktose in 4 Stunden 2,Io $\mathrm{g}$ ausgeschieden. Die Pat. starb I3 Monate nach der Operation und mußte alle 2 Wochen punktiert werden.

II. Gruppe: Omentofixation in eine präperitoneale Tasche. (Fälle 3 bis inkl. 6.)

Hier wurde so vorgegangen, daß nach der Laparotomie das Peritoneum der vorderen Bauchwand von der Schnittlinie aus beiderseits abgelöst und in die so gebildeten Taschen Neizzipfel eingenäht wurden.

Fall Nr. 3 machte eine $1 / 2$ stündige Billrothmischungsnarkose durch. Bei ihm zeigte der weitere Verlauf eine vollständige 
Darmparalyse und die nach 5 Tagen post operationem ausgeführte Obduktion eine Degeneration des Herzfleisches. Da in diesem Falle cin Ikterus gravis bestand und reichlich Blut sich in Magen und Darm vorfand, muß auch der Cholämie eine Hauptschuld gegeben werden.

Krankengeschichte:

K., Karl, 50 jähr. Obermaschinist, akquirierte 1878 in Bosnien Malaria. Vor 3 Jahren Gallensteinkoliken und Ikterus. Starker Trinker. Spitalaufenthalt vom 4. V. bis 18 . V. 1907.

Aus dem Stat us praesens: Kräftig gebauter, fetter Mann; starker Ikterus, hochgradiger Ascites. Druckempfindlichkeit der Lebergegend. Große Hämorrhoidalknoten.

Operation am ir. V. Leber verkleinert, derb, Oberfläche höckerig. In der Gallenblase Gallensteine. Pacquelinisierung der Hämorrhoiden. Stopfrohr. Mittel.

15. V. Kein Stuhl trotz aller Bemühung und aller angewandten

I6. V. Zunehmende Somnolenz. Puls 130. Pat. reagiert kaum auf Anruf. Kein Stuhl. Exitus am I8. V.

Obduktionsbefund: Zirrhose der Leber, Ikterus gravis. Chron. Milztumor. Blutung in den Magen und Darm (aus Ösophagusvaricen?). Zcigefingerdicke Sappeysche Vene. Chron. Magen- und I)armkatarrh. Degeneration des Herzfleisches. Cholelithiasis.

Fall 4. Tuberkulöse Peritonitis mit Ascites. Die Leber erwies sich stark zirrhotisch verändert. Der Eingriff wurde in Lokalanästhesie ausgeführt und besserte den Zustand der Kranken sehr wesentlich. Sic ist jetzt, seit September I 91 I, vollkommen arbeitsfähig.

Krankengeschichte:

M. O., 46 jähr. Private aus Wien. Vor 7 Jahren Gelenkrheumatismus. Im Juli 1909 plötzlich an Schmerzen im Rücken und in der IIerzgegend erkrankt, zunchmende Atembeschwerden, Ödeme der Beine und Anschwellen des Bauches.

Aus dem Status praesens: Mittelgroße Frau in gutem Ernährungszustand. Ödeme. Ascites. Im Sputum keine Tuberkelbazillen. Calmette -t-t. GroBe derbe Leber. Wassermann negativ. Auf Galaktose per os $(40 \mathrm{~g})$ werden in 3 Stunden $0,4 \mathrm{~g}$ im Harne ausgeschicden. Bauchumfang $\mathrm{I} I \mathrm{~cm}$. Unter der Annahme einer Concretio cordis wurde an einer anderen Station ein Stück aus der 4., 5. und 6. Rippe reseziert. Bei der Operation zeigte sich, daß keine Concretio bestehe (II. I9IO). Keine Besserung post op. 
Bald darauf 2 mal Haematemesis, Erbrechen; Zunehmen des Bauchumfangs. Häufige Punktionen (Punktat: sp. Gew. IO20, reichlich Lymphocyten enthaltend und grampositive Diplokokken). März I9I I: Galaktoseversuch (40 g), in 3 Stunden werden 2,207 $\mathrm{g}$ ausgeschieden. Juni I9II: Bauchumfang $135 \mathrm{~cm}$. Da trotz reichlicher Punktionen und Anwendung aller sonstigen Heilmittel die Beschwerden von seiten des Ascites stetig zunehmen, wird am 4. IX. I9I I die Operation vorgenommen. Es entleerten sich dabei 25 Liter Ascitesflüssigkeit. Die I,eber war stark zirrhotisch verändert.

Seit der Operation fühlt sich die Pat. sehr erleichtert. Sie wurde seither nur 2 mal punktiert. Kein Anschwellen der Beine mehr. Pat. ist wieder arbeitsfähig.

Fall 5 litt seit 4 Jahren an einer Concretio pericardii cum corde und einer Mitralinsuffizienz und -stenose. Bei der in Lokalanästhesie ausgeführten Operation zeigte sich die Leber klein und zirrhotisch. Das Netz war stark verdickt und bereits mit dem Peritoneum parietale verwachsen. Wie nicht anders zu erwarten war, trat hier keine wesentliche Besserung im Befinden der Kranken ein. Dieser Fall war offenbar für eine Operation vollständig ungeeignet.

Krankengeschichte:

E. M., 2 I jähr. Mädchen. Mit Iı Jahren Gelenkrheumatismus, 2 Jahre später Pericarditis, 3 Jahre darauf Rippenfellentzündung rechts; seither stets Herzklopfen, Kurzatmigkeit, Anschwellen der Beine, der Lider; häufiger Spitalaufenthalt. Starker Ascites, der oft punktiert wurde.

Aus dem Stat us praesen s: Im Harne kein Albumen. Wassermann negativ. In der Ascitesflüssigkeit I4 Prom. Eiwciß, spez. Gew. IoIo; im Sediment vorwiegend Leukocyten. Galaktoseversuch negativ.

Operation 4. IX. IgI in Lokalanästhesie. Abfluß von I 4 Liter klarer Flüssigkeit. Leber klein, zirrhotisch. Netz stark verdickt, mit Pcriton, verwachsen. Heilung p. pr. Der Ascites kehrte bald wieder und mußte seither häufig punktiert werden.

Fall 6 verdient gewiß eine ganz besondere Beachtung. Hier wurde auf den Vorschlag des Herrn Privatdozenten Dr. Porges bei einer Frau, welche seit drei Jahren wegen starken Ascites bei Trikuspidalinsuffizienz fast stets bettlägerig war und bei welcher früher sehr zahlreiche Punktionen ausgeführt werden mußten, der Versuch einer chirurgischen Beeinflussung vorgenommen. 
Der Erfolg war ein eklatanter. Die Patientin berichtete uns kürzlich aus ihrer Heimat, daß sie jetzt umhergehe und daß ihr Allgemeinzustand sich wesentlich verbessert habe. Sie wurde kurz nach der Operation ein einziges Mal punktiert und ist jetzt frei von Ascites und arbeitsfähig ( 8 Monate post operationem).

Krankengeschichte:

H. M., 38 jähr. Taglöhnersgattin aus Galizien, war bis zum 35. Lebensjahr gesund. Seither die Herzbeschwerden. Seit 3 Jahren Ascites, weswegen sie sehr oft punktiert werden muß. Da der Bauch sehr bald groß und schwer wird, muß die Kranke fast stets im Bette liegen. Spitalaufenthalt vom 13. VII. bis I3. VIII. I9I2.

Aus dem $S$ tatus praesens: Sehr starker Ascites. Punktion. (Das Punktat enthält 4,206 Proz. Eiweiß, spez. Gew. 10I8.) Positiver Leberpuls. Mitral- und Tricuspidalinsuffizienz. Keine Ödeme der unteren Extremitäten.

Operation am I8. VII. I9I2 in Lokalanästhesie. Bauchdecken kolossal rarefiziert. Netz stark geschrumpft. Leber groß, weich, rotbraun. Leberpuls. Peritoneum sehr brüchig und von kautschukähnlicher Konsistenz. Heilung p. pr. Seither Wohlbefinden.

III. Gruppe: Omentofixation in eine subkutane Tasche nach Narath. (Fall 7 und 8.)

Nr. 7. Alkoholische Zirrhose mit Ascites. Der Operationsbefund ergibt eine kleine granulierte Leber. Das Netz war geschrumpft, seine Gefäße ektatisch. Dieser Fall gehört zu denjenigen, welche man anführen muß, wenn man auf die Wichtigkeit einer besonders sorgfältigen Bauchdeckennaht aufmerksam machen will. Denn hier starb der Patient an einer Peritonitis, die durch eine Nahtdehiszenz entstanden war, aus welcher ständig Ascitesflüssigkeit ausfloß und den Kranken durchnäßte.

Krankengeschichte:

P. L., 57 jähr. Taglöhner aus Görz. Seit Juni I910 Schwellung der unteren Extremitäten; bald darauf Ascites. Keine Magendarmbeschwerden. Potus I Liter Wein, 8 Krügel Bier tägl. Lues seit I 89r.

Aus dem Status praesens: Großer, kräftiger Mann, Ödeme der unteren Extremitäten, Ikterus. Bauchdecken gespannt, keine Venenektasien, starker Ascites, Druckempfindlichkeit in der Lebergegend, Milztumor. Im Harne 5 Prom. Albumen bis I2 Prom. abwechselnd. Pat. wurde sehr oft punktiert.

Wird mit der Diagnose Leberzirrhose, Nephritis am 22. III. I9I I operiert. Lokalanästhesie. Leber klein, granuliert. Netz ge- 
schrumpft. Am 27. III. entleert sich aus einer Nahtdehiszenz reichlich Ascitesflüssigkeit. Von nun ab liegt Pat. in einem stets durchnäßten Bette. Am 3. IV. heftige Schmerzen im Abdomen, Puls sehr frequent, Zunge trocken, peritoneale Reizerscheinungen. 5. IV. Exitus.

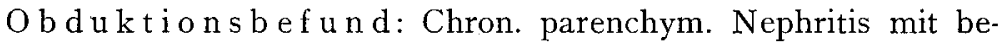
ginnender Schrumpfung der linken Niere. Serös-fibrinöse Peritonitis mit reichlichem Exsudat. Hepar lobatum. Akutes Lungenödem; chron. Emphysem; Stauungsorgane.

Fall 8 betrifft eine alkoholische Zirrhose, wo der Ascites erst seit zwei Monaten bestand. Hier fand sich ebenfalls das Netz mit dem Peritoneum parietale verwachsen vor. Es wurde der Patient nach der Operation noch dreimal punktiert. Nunmehr ist er vollständig von Ascites geheilt. Dieser Fall gehört zu unseren besten Erfolgen und scheint für die Überlegenheit der subkutanen Einnähung des Netzes zu sprechen. Denn die Besserung trat ein, wiewohl früher Netz mit dem Peritoneum parietale verwachsen waren.

Krankengeschichte:

F. L., 52 jähr. Tischler aus Mähren, wurde im Jahre 1907 an unserer Klinik wegen Stenosis pylori post ulcus ventriculi operiert (Gastroenteroanastomose). Schon damals verzeichnete der Operationsbefund zirrhotische Leber. Der Ascites trat erst 4 Jahre später auf, 2 Monate vor der Operation. Pat. ist mäßiger Potator.

Aus dem Status praesens: Magerer, schwächlicher Mann, Ödeme der unteren Extremitäten; Abdomen stark aufgetrieben, dessen Hautvenen ektatisch, starker Ascites. Milztumor. In der Ascitesflüssigkeit 6 Prom. Albumen, spez. Gew. Ioo8. Galaktoseprobe (40 g per os). In 6 Stunden wurden $2,7 \mathrm{~g}$ Galaktose ausgeschieden.

Operation am 17. X. I9I i in Lokalanästhesie. Netz auf ca. 5 Kronenstückgröße am Peritoneum angewachsen vorgefunden. Omentopexie in eine subkutane Tasche. Wundheilung p. pr. Post op. werden von $40 \mathrm{~g}$ Galaktose in 4 Stunden 3, I g ausgeschieden. Nach wenigen Punktionen bleibt der Ascites dauernd aus.

IV. Gruppe: Präperitoneale Fixation der Milz plus Omentopexie (Narath). (Fälle 9 bis inkl. I2.)

Bei der Einnähung der Milz wurde diese durch Abreiben mit Jodtinktur ,angefrischt" und zwischen Peritoneum und Muskulatur eingenäht.

Fall 9 war ein Potator strenuus ( 5 Liter Wein täglich), der seit einem Jahre bereits ikterisch war. Nach der Operation hat 
der Mann seine volle Arbeitsfähigkeit wieder erlangt. Es trat kein Ascites mehr bei ihm auf. Er starb nach Mitteilung seiner Verwandten 2 Jahre post operationem an „Herzschlag“.

Dieser Fall muß, wiewohl er als ein sehr aussichtsloser für die Operation gelten mußte, als ein in jeder Weise befriedigender angesehen werden.

Krankengeschichte:

J. P., 36 jähr. Sattler, war bis Mai I905 gesund. I8-20 Viertel Wein täglich. Erkrankt an Gelbsucht, bald darauf Anschwellen der Beine, Abmagerung, Bauchwassersucht, starke Schmerzen in den Flanken.

Aus dem Status pra esens: Starker Ikterus, Abdomen stark aufgetrieben. Ascites. Leber und Milz vergrößert. Kleine Ventralhernie. Diagnose: Leberzirrhose.

Pat. wird am 17. I. Igo6 operiert. Die Leber ist derb, ihre Oberfläche granuliert. Anfrischung der Milzkapsel und Einnähung derselben und eines Netzzipfels zwischen Peritoneum und Muskulatur. Wundheilung p. pr. Pat. wird bald geheilt entlassen und bleibt bis zu seinem Tode (er stirbt 2 Jahre nachher an „Herzschlag") beschwerdefrei.

Fall ro, schwerer Trinker, leidet seit 3 Jahren an Leberzirrhose. Wird in Narkose operiert, wiewohl er eine schwere Albuminurie aufweist. Hier wurde das Cavum peritonei drainiert. Der Obduktionsbefund verzeichnet eine schwere parenchymatöshämorrhagische Nephritis, eine Concretio cordis und beginnende Pneumonie. Daß dieser Fall statistisch unter die Mißerfolge einzureihen wäre, die der Operation zur Last fallen, wird niemand behaupten. Er müßte bei der prozentuellen Berechnung der Resultate unberücksichtigt bleiben.

Krankengeschichte:

46 jähr. Selcher; vor 7 Jahren Gelenkrheumatismus. Vor 3 Jahren plötzliches Auftreten von Ödemen an den Beinen, die unter ärztlicher Behandlung schwinden. Nach einigen Monaten wieder Ödeme, Ascites, Atembeschwerden. Zahlreiche Punktionen.

Aus dem Status praesens: Stark abgemagerter Mann, Herzdämpfung verbreitert, Zwerchfellhochstand. Abdomen stark aufgetrieben, tonnenartig, Bauchhaut gespannt, glänzend. Venen ektatisch. Ödeme der unteren Extremitäten. Im Harne Eiweiß.

Operation am 28. VI. 1907 in Billrothmischung-Narkose. Das Netz wird an einer Stelle der vorderen Bauchwand in Hand- 
tellergröße adhärent vorgefunden. Am unteren Wundrand wird ein Gazestreifen zur Ilerausleitung des Ascites verwendet. 29. VI. Starker Abfluß von Ascitesflüssigkeit. 30. VI. Subjektives Wohlbefinden. Beim Verbandwechsel am Abend ist Pat. stark benommen, der Puls sehr frequent. Es tritt ein soporöser Zustand ein, der Puls wird kaum fühlbar; am 3. VII. Exitus.

O bduktions b ef und: Atrophische Leberzirrhose, chron. Perihepatitis, l'erisplenitis, Verdickung des Peritoneums und des Netzes. Parenchymatös hämorrhagische Nephritis, Concretio cordis cum pericardio, fettige Degeneration des Herzfleisches, Lungenödem. Beginnende Pneumonie im rechten Unterlappen.

Im folgenden Falle Nr. I I setzte eine interkurrent aufgetretene hämorrhagisch fibrinöse Pericarditis $4^{1} / 2$. Monate post operationem dem Leben der Patientin ein Ende.

Krankengeschichte:

M. M., 50 jähr. Kräutlerin, hat vor 3 Jahren einige Tage lang Blut gespuckt. Ende November 1909 schwellen die Beine an; bald darauf auch Bauchwassersucht. Seit Mai 1910 ist Pat. bettlägerig. Vor einem Monat trat Gelbsucht auf. Pat. hat seit Kindheit viel Bier getrunken. Seit Io Jahren $1 / 2$ Liter Wein täglich.

Aus dem Status praesens: Starker Ikterus. Hochstand des Zwerchfells. Bauchumfang $127 \mathrm{~cm}$. Bedeutender Ascites. Nabel vorgetrieben. Venennetz der Bauchhaut deutlich ausgebildet. Nach Punktion des Abdomens wird die Leber 2 Querfinger unter dem Rippenbogen tastbar; sie ist hart, Oberfläche granuliert. Milztumor. Das Punktat gelblich-grün. Eiweiß I3 Prom., spez. Gew. Iori.

Pat. wird am 6. VII. in Totalanästhesie operiert. I)as Netz war stark vaskularisiert und verdickt. Die Milz vergrößert, ihre Kapsel verdickt. - Die Heilung erfolgte p. pr. Die Frau wurde in ambulatorische Behandlung entlassen, mußte noch einige Male punktiert werden, starb aber an einer interkurrenten I'erikarditis, $4^{1 / 2}$ Monate nach dem Eingriff.

Obduktionsbefund: Atrophische Leberzirrhose, chron. Milztumor; Stauungskatarrh in Magen und 1)arm. Perisplenitis und Perihepatitis fibrosa, Hydrothorax. Anasarka. Hämorrhagisch-fibrinöse Perikarditis. Stauungsorgane.

Fall 12 betrifft ein jetzt 23jähriges Mädchen, bei welchem wegen bestehenden Ascites im Jahre I 907 die Omentofixation ausgeführt wurde. Da keine Besserung eintrat, fügte man ein Jahr später die subkutane Einnähung der Milz hinzu. Es handelte sich hier um einen Morbus Curschmann (Polyserositis), ein Fall, der noch späterhin der Gegenstand weiterer Eingriffe wurde und 
dessen Krankengeschichte sich Herr Prof. Pàl zur Bearbeitung vorbehalten hat. Dieser Patientin brachte eine Cardiolyse eine wesentliche Besserung.

V. Gruppe: Omentopexie in eine präperitoneale Tasche plus Annähung des linken Leberlappens. (Fall I3.)

Fall 13 war ein schwerer Potator (3 Liter Wein täglich); es bestand bei ihm ein systolisches Geräusch über der Aorta, Verbreiterung der Herzdämpfung und sonstige Symptome allgemeiner Arteriosklerose. Der Mann wurde in $1 \frac{1}{4}$ Stunden lang andauernder Billrothmischungsnarkose ( $150 \mathrm{~g}$ ) operiert. Er verließ in gebessertem Zustand dic Klinik, wurde in der Folgezeit noch viermal punktiert und starb 3 Monate post operationem in seiner Heimat.

Krankengeschichte:

F. Z., 53 jähr. Wirt aus Mähren. Wiederholt Gelenkentzündungen. Seit November 1905 Magendarmbeschwerden und Zunahme des Bauchumfanges. Ödeme an den Beinen und am Scrotum. Zahlreiche Funktionen vor der Operation. Potus I2 Viertel Wein täglich, außerdem Bier und Schnaps.

Aus dem Status praesens: Verfallen aussehender Mann, Gesichtsfarbe fahl. Haut mager, welk. Herzdämpfung verbreitert, systolisches Geräusch über der Aorta. Radialis starr, geschlängelt. Hochstand des Zwerchfells. Abdomen kugelig vorgetrieben, Bauchhaut gespannt, von großen ektatischen Venen durchzogen.

Operation am 3. II. I906. Linker Leberlappen bedeutend vergrößert. Leber fein granuliert, derb. Milz nicht wesentlich vergrößert. Wundheilung p. pr. Am 20. II. wird Pat. ohne Ascites entlassen, wurde noch 4 mal punktiert und starb nach 3 Monaten.

VI. Gruppe: Omentopexie plus beiderseitige Saphenopexic. (Fall I 4.)

Bei der Saphenopexie wurde folgendermaßen vorgegangen: Die Vena saphena wurde rechts unterbunden (etwa $8 \mathrm{~cm}$ von ihrer Einmündung in die Vena femoralis) und durchtrennt. Darauf wurde der proximale Stumpf durch ein Magnesiumröhrchen durchgezogen, über den Rand desselben gestülpt und fixiert. Das so von Intima überzogene Röhrchen wurde dann ins Peritoneum eingenäht, so da $B$ Intima und Serosa aneinander lagen. 9 Tage später wurde das gleiche Verfahren links angewendet und die Omentopexie hinzugefügt. 
Krankengeschichte:

E. S., 44 jähr. Kutschersgattin. Leidet seit 6 Jahren an Atembeschwerden beim Stiegensteigen und bei Anstrengungen. Seit März I9I I Größerwerden des Bauches, Schmerzen in der Oberbauchgegend, Ikterus. In der letzten Zeit stark abgemagert.

Aus dem Status praesens: Ikterische kleine Frau, Ödeme der unteren Extremitäten, Herzdämpfung verbreitert; Abdomen kugelförmig vorgetrieben. Größte Zirkumferenz Io6 $\mathrm{cm}$. Nach Entleerung des Ascites durch Punktion wird die Leber in Nabelhöhe getastet, sie ist von derber Konsistenz, ihre Oberfläche grobhöckerig. Im Punktat Io Prom. Eiweiß, spez. Gew. 10o8. Im Harne Albumen, Zylinder und Eiter. Die Pat. wurde sehr oft punktiert.

Am 28. XI. und 7. XII. 19I I beiderseitige Saphenopexie und Omentopexie.

Die Pat. starb am 29. XII. unter zunehmendem Ascites; sie hatte in der letzten Zeit subfebrile Temperaturen und I20-I34 Pulsschläge in der Minute.

Die Obduktion ergab folgenden Befund: Atrophische Leberzirrhose, chronischer Milztumor mit subakuter Schwellung. Ascites, Hydrothorax, ödematöse Schwellung der Magen- und Darmschleimhaut. Akute und subakute Miliartuberkulose im Mediastinum und Peritoneum. Cholelithiasis. Marasmus.

Im vorstehenden haben wir die Fälle nach den in Anwendung gebrachten Operationen geordnet; wir meinen aber, $\mathrm{da} B$ es in diesem Gegenstande nicht so sehr auf die Operationsmethode als vor allem auf die geeigneten Fälle und den richtigen Moment zum Eingreifen ankommt. Daß der Auswahl der Operation auch eine Bedeutung zufällt, darüber gibt es wohl keinen $Z$ weifel; aber diese Auswahl wird meist intra operationem, je nach dem Befunde, zu treffen sein. So wird man sich z. B. keinen Vorteil von einer einfachen Omentopexie versprechen dürfen, wenn, wie dies nicht gar selten beobachtet wird, das Netz an der vorderen Bauchwand bereits fixiert vorgefunden wird, oder wenn es stark geschrumpft ist.

Ordnen wir unsere Fälle nach den Krankheitszuständen ein bzw. nach der klinischen Diagnose, dann ergeben sich:

5 Fälle von alkoholischer Leberzirrhose,

2 ", infektiöser bzw. bakteriotoxischer Leberzirrhose,

2 ," , Tuberkulose des Bauchfells,

2 " " Concretio cordis, 
I Fall von Stauungsleber,

I ", , Tricuspidalinsuffizienz.

Wie waren die Aussichten auf Erfolg bei den einzelnen Fällen und welches war ihr weiterer Verlauf? Was die alkoholische Zirrhose der Leber betrifft, so sind sich die Autoren darüber im klaren, daß die unkomplizierte Zirrhose die besten Chancen für die Heilung des Ascites bietet. Von unseren Fällen können leider nur zwei als unkomplizierte betrachtet werden (Nr. 8 u. 9), wobei der eine dieser Kranken seit Monaten bereits einen starken Ikterus aufzuweisen hatte. Von den vier anderen Zirrhotikern wies der eine (Nr. Io) bei der Obduktion eine Concretio cordis und eine parenchymatös-hämorrhagische Nephritis auf; Fall I 3 hatte eine schwere Arteriosklerose. Von den beiden restlichen war der eine, Fall I4, durch Tuberkulose des Bauchfells, der zweite, Nr. 7, durch Lebersyphilis kompliziert. Ob nun deswegen so selten unkomplizierte Zirrhosen zur Operation gelangten, weil sie nicht früh genug in Beobachtung kamen (so lange sie nämlich unkompliziert waren) oder ob die zuweisenden internen Stationen das Eingreifen in diesem Stadium als unnötig erachteten und die Operation nur als ultimum refugium bei sonst verlorenen Kranken gelten lassen - Tatsache ist, daß auch in unseren unkomplizierten Zirrhosefällen die besten Resultate aufzuweisen waren: beide Fälle blieben von Ascites dauernd geheilt. Während wir den einen der vier anderen Fälle infolge seiner durch Nahtdiastase und Fistelbildung veranlaßten Infektion des Bauchfells verloren haben, läßt sich bei den übrigen Fällen mit vollem Recht sagen, daß sie an den Komplikationen ihrer Zirrhose gestorben sind. Bei unseren zwei wegen Bauchfelltuberkulose operierten Patientinnen brachte die Operation das eine Mal (Nr. 4) das Verschwinden des Ascites. Das andere Mal (Nr. 2) trat er zwar wieder auf und mußte bis zum Lebensende regelmäßig durch Paracentese entleert werden, doch war dies viel seltener der Fall als vor der Operation. Bei der Patientin, an welcher die Saphenopexie ausgeführt wurde (Fall I4), fand sich bei der Obduktion neben der Leberzirrhose, wegen welcher sie zur Operation bestimmt worden war, eine Miliartuberkulose des Bauchfells. Ob hier durch die Saphenopexie nicht eine Dissemination der Tuberkulose durch Einströmen der tuberkulösen Ascitesflüssigkeit in die Blutbahn 
bewirkt wurde, könnte man nicht ohne Grund fragen. Unter derartigen Umständen würde die Ausführung der Ruotteschen Ope. ration eine eminente Lebensgefährdung bedeuten. Diese Überlegung diene uns zur Warnung vor diesem Eingriffe bei Kranken, wo wir eine Bauchfelltuberkulose oder -carcinose nicht mit voller Sicherheit ausschließen können.

Mit der Patientin M. M. (Nr. I I), bei welcher wegen bestehender Stauungsleber chirurgisch eingegriffen wurde, hatten wir insofern wenig Glück, als sie uns bereits mit starken allgemeinen Ödemen und einem derartigen Daniederliegen der Herztätigkeit zugewiesen wurde, daß kein rechter Erfolg erwartet werden konnte. Der Ascites trat langsam wieder auf; doch starb die Frau an einer hämorrhagisch-fibrinösen Pericarditis $4 \frac{1}{2}$ Monate post operationem.

Auch beide Fälle von Concretio cordis hatten keinen Vorteil von der Operation. In dem einen Falle (Nr. 5) rezidivierte der Ascites -- vielleicht weil ein schweres Vitium nebenbei bestand; in dem zweiten, Nr. I 2, rezidivierte er ebenfalls, obwohl hier das Vitium fehlte. Wenn auch diese beiden Fälle die Frage nach der Berechtigung der Talmaschen Operation bei der Concretio cordis natürlicherweise nicht $\mathrm{zu}$ beantworten vermögen, so könnten sie jedenfảlls bei einer eventuellen Zusammenstellung derartiger Fälle als erfolglos Operierte gezählt werden.

Tricuspidalinsuffizienz: Dieser Klappenfehler nimmt bekanntlich eine außergewöhnliche Stellung ein in bezug auf das Verhältnis zwischen Ascites und Ödemen der unteren Extremitäten. Ich habe vorhin schon erwähnt, daß wir bei der Patientin, die an einer Mitral- und Tricuspidalinsuffizienz litt, Nr. 6, einen ganz unverhofft günstigen Exfolg aufzuweisen hatten.

Überblicken wir unser Material, so zeigt sich, daß von I4 operierten Patienten 8 mit der Diagnose „Leberzirrhose, Ascites" zur Operation bestimmt wurden. Die Autopsie in vivo und in mortuo und der weitere Verlauf ergaben jedoch, daß es sich in einer ganz geringen Zahl der Fälle um unkomplizierte Zirrhosen gehandelt hatte. Es wurde daher oft, zum Teil trotz vorhandener Kontraindikationen, zum Teil unter einer falschen Voraussetzung, operiert: wenn auch bei der tuberkulösen Peri- 
tonitis ein Eingriff meistens nur von Nutzen sein kann, so muß man doch bedenken, wie verschieden die prognostischen Erwägungen dabei sind. Von den Mißerfolgen der Operation bei chronisch-entzündlich bedingtem Ascites frappiert, riet Lieblein, eine Operation nur dann auszuführen, wenn reine Stauung besteht. Bei Lues hepatis und chronischer Peritonitis könne kein günstiger Erfolg erwartet werden, weshalb er empfiehlt, stets das spezifische Gewicht bzw. den Eiweißgehalt der Ascitesflüssigkeit zu bestimmen und ihn zur Entscheidung zu verwerten, ob es sich um einen entzündlichen oder einen Stauungserguß handelt. Nun aber muß bedacht werden, wie aussichtslos oft die Bemühung ist, den Ascites als Exsudat oder als Transsudat zu erkennen.

Ich weise diesbezüglich auf die Äußerung Talmas hin, daß die Vorstellung falsch sei, als könne man durch das spezifische Gewicht die Differentialdiagnose zwischen Transsudat und Exsudat stellen in dem Sinne, daß ein Gewicht niedriger als Io 4 gegen die entzündliche Natur des ursächlichen Prozesses sprechen sollte. Chauffard (36) führt als untere Grenze für entzündlich bedingten Ascites das spezifische Gewicht Ior6 an, ReuB IoI8. Wie man sieht, schwanken die Grenzen sehr bedeutend. Wenn man weiter bedenkt, daß sehr oft, insbesondere nach Punktionen, sich Inflammationen in der Peritonealhöhle von Ascitikern abspielen, wenn man an den von Talma so ausführlich besprochenen Symptomenkomplex von Peritonitis serosa mit meist sekundärer Leberzirrhose denkt und schließlich bedenkt, wie häufig sich tuberkulöse Peritonitiden mit Stauungsascites kombinieren, dann ist die Bemühung, die Ätiologie des Ergusses stets einwandfrei aus dessen Eiweißgehalt aufzuklären, illusorisch. Da bekanntlich auch oft die chemische sowie die mikroskopische Untersuchung des Punktates im Stich läßt, ja zuweilen zu Trugschlüssen veranlaßt, dann kann leider der gute Rat Liebleins (37) nicht immer richtunggebend sein.

Worin bestehen die Vorteile, die wir von einem operativen Eingreifen erwarten?

I. Die momentane Behebung des Ascites und in geeigneten Fällen die dauernde Beseitigung desselben. 
2. Hebung des Allgemeinbefindens durch Verminderung des intraabdominellen Druckes, durch Befreiung des Zwerchfells und somit der Atmung; Besserung der Blutzirkulation.

3. Besserung der Leberfunktion (Axis a [38]).

4. Verifizierung der Diagnose durch Autopsia in vivo.

Der letzte Punkt erscheint uns besonders wert, hervorgehoben zu werden. Denn gerade bei den Fällen, die mit Ascites einhergehen, sind die diagnostischen Erwägungen und Befunde sehr erschwert.

Wir müssen vor allem bedenken, daß die Erkennung der Leberzirrhose in ihrem Anfang sehr viel Aufmerksamkeit verlangt. Daß man so früh als möglich zur Operation zu schreiten hat, will man in wirkungsvoller Weise gleich zu Anfang dem Ascites entgegentreten, darüber wird nicht mehr viel debattiert.

In seinem Resumé sagt Koch (loco cit.) sogar: „Die Operation ist bei weit fortgeschrittenen Fällen gefährlich und soll deshalb in frühen Stadien der Krankheit gemacht werden." Woran aber soll man die frühen Stadien erkennen?

Im folgenden will ich mir erlauben, diese Frage kurz zu beantworten und auf die Anfangssymptome der Leberzirrhose hinzuweisen. Die Autoren geben als Symptome beginnender Leberzirrhose eine Anzahl von Zeichen an, die aber nicht immer und in ihrer Gesamtheit anzutreffen sind. Zu den ersten Anzeichen zählt man das präascitische Ödem. Chauffard beschreibt es folgendermaßen: „Ein fast vereinsamt dastehendes und initiales Symptom der beginnenden Leberzirrhose ist das präascitische Ödem. Es handelt sich um ein weißes indolentes Ödem, das sich ziemlich leicht wegdrücken läßt und das symmetrisch an beiden Fußknöcheln beginnt, um dann zuweilen beide Beine oder sogar die subdiaphragmatische Körperhälfte zu ergreifen. Diese Ausbreitung weist darauf hin, daß es mit einẹ mehr oder minder vollständigen Zirkulationsstörung in der unteren Hohlvene in ursächlichem Zusammenhang steht, für welche die durch die Zirrhose hervorgerufenen Schädigungen der Venen verantwortlich sind. Das präascitische Ödem kann sehr frühzeitig auftreten, lange vor den ersten Allgemeinstörungen der Leberzirrhose; es kann der Ascitesbildung einige Monate, ja ein bis anderthalb Jahre vorausgehen; doch begleitet es in der 
Regel die ersten Anzeichen der Zirrhose und beansprucht daher einen großen semiologischen Wert."

Von vielen Autoren wird als Frühsymptom das Auftreten eines Milztumors aufgefaßt. Während der Milztumor bei der Leberzirrhose meist lediglich als Folge der Blutstauung im Pfortadergebiete angesehen wurde, hält man ihn jetzt ,,entweder für den Ausdruck einer gleichwertigen Erkrankung aus derselben Ursache oder sogar für das primäre Leiden" (Hoppe-Seyler [39] in Nothnagels Handbuch I9I3).

Auf die im Verlaufe einer Leberzirrhose auftretenden Blutungen ist von vielen Seiten als auf ein initiales Symptom hingewiesen worden. Chauffard bezeichnet Nasenbluten als eines der häufigsten Anzeichen. Pel erklärt als wichtige Erfahrung, $\mathrm{da} B$ die Blutungen, die, wie wir seit Litten (40) wissen, durch Ruptur variköser Venenerweiterungen im untersten Teile der Speiseröhre zustande kommen, daß diese Blutungen das allererste Symptom des Leberleidens sein können. Wenn man bedenkt, $\mathrm{da \beta}$ in etwa $1 / 3$ der Fälle die Blutungen von vornherein tödlich sind und da $\beta$ sie in den restlichen Fällen periodisch immer wieder auftreten (Preble, zit. in Bunge), dann muB man sich darüber wundern, wie verhältnismäßig wenig man in den Krankengeschichten über Blutungen bei Zirrhotikern liest. Das ist wohl darauf zurückzuführen, daB es sich in den periodisch auftretenden Blutungen oft nur um kleine kapilläre Hämorrhagien handelt, wo nur ganz geringe Blutmengen ausgespuckt werden. Pel vergleicht diese Blutungen mit den initialen Hämoptöen bei Lungentuberkulose und spricht von initialer Hämatomesis bei Leberzirrhose. Lange (4I) fand unter 56 sezierten Fällen von Leberzirrhose Iomal Blutungen, und zwar in 37 Proz. der hochgradigen Fälle, gar nicht bei beginnender Zirrhose. Durch diese Befunde wird jedoch die Wichtigkeit der initialen Blutungen bei dieser Erkrankung keineswegs abgeschwächt.

Gelegentlich der Diskussion über den Vortrag von Sinclair White, der die chirurgische Behandlung des Ascites betraf (Brit. med. Journ. I 906) sagte W. J. Ma yo: „Es scheint mir, als seien wir in diesem Gegenstand vom klinischen Standpunkt so weit als möglich gegangen. Nun müssen wir unsere Kenntnisse von den Leberfunktionen erweitern, um das Problem der Lösung zuzu- 
führen." Die Klagen, daß unsere Kenntnisse von den Leberfunktionen noch nicht genügend aufgebaut seien und die Forderung nach klinisch verwertbaren Leberfunktionsprüfungsmethoden kehren immer wieder. „Die Ausbeute an diesen Methoden," sagt Ewald, ,ist trotz vielfältiger und mühevoller Versuche nicht gerade erheblich; wir besitzen zwar eine Anzahl von Methoden, die uns über die Leistungen des Organs im großen und ganzen unterrichten, aber keine derselben reicht aus, uns das Vorhandensein eines bestimmten Krankheitsprozesses mit Sicherheit erkennen zu lassen." In seiner Arbeit ,zur Frage der Talmaschen Operation" beklagt sich auch Goetjes (43) darüber, daß wir keine quantitativen Leberfunktionsprüfungsmethoden besäßen. Wenn wir nun auch nicht mit mathematischer Präzision die Lebererkrankungen zu diagnostizieren vermögen, so ist es dennoch mit den funktionellen Prüfungsmethoden nicht so schlecht bestellt. Gewiß wird der Wert der einen oder der anderen Methode nicht in allen Punkten von der Mehrzahl der Autoren anerkannt; doch die uns bekannten Verfahren dürften uns in ihrer Gemeinsamkeit gewiß wertvolle Aufschlüsse über den jeweiligen Zustand der Leberzellen und über die Aussichten einer Intervention geben. Aus der Menge der funktionellen Anhaltspunkte, welche uns eine Schädigung der Leberzelle anzeigen, seien die anerkanntesten hervorgehoben: Von französischen Autoren wird auf die verspätete Harnausscheidung Gewicht gelegt. Während der Gesunde 1/2 Liter Wasser innerhalb der folgenden sechs Stunden in der 2. bis 3 . Stunde ausscheidet, findet man bei Leberkranken, speziell bei Pfortaderstauung die Wasserausscheidung verspätet, in der Hauptsache erst in der 4. bis 5. Stunde kommend (Gilbert et Lereboullet [44] „L'opsiurie dans les maladies du foie“). Diese Prüfung auf „Opsiurie“ ist klinisch leicht ausführbar. Nicht schwerer durchführbar sind die chemischen Funktionsprüfungsmethoden, von denen ich die Untersuchung auf

1. verminderte Harnstoffausscheidung,

2. Urobilinurie,

3. alimentäre Lävulosurie und

4. alimentäre Galaktosurie anführen will.

Während die Ansichten über den diagnostischen Wert der 
Harnstoff- und Ammoniakausscheidungsverhältnisse bei Leberkranken stark auseinandergehen, scheint man der von GläBner (45) vorgeschlagenen Prüfungsmethode mehr Vertrauen entgegen zu bringen. Dieser wies nämlich nach, daß bei den das Leberparenchym destruierenden Prozessen bestimmte Mengen verfütterter Aminosäuren (Alanin, Glykokoll, Asparaginsäure) nicht vollständig in Harnstoff übergeführt werden können, sondern zum Teil als solche wieder ausgeschieden werden. Im Gegensatz dazu ist die normale Leber imstande, große Mengen dieser Substanzen zu verarbeiten. Ein Leberzirrhotiker scheidet z. B. von 20 bis $30 \mathrm{~g}$ per os genommener Aminosäuren 50-70 Proz. unverändert aus.

Wie wichtig die Feststellung der Urobilinurie bei der Leberzirrhose ist, zeigt der Ausspruch Pels: „Ich halte dieses Zeichen für das Erkennen der Leberzirrhose so wichtig, daß ich zögere, die Diagnose zu stellen, wenn diese Urobilinurie fehlt." Ebstein (46) (Handbuch d. prakt. Med.) sagt: „Ich möchte beim Fehlen von starkem Urobilingehalt bei gleichzeitigem Ascites die atrophische Leberzirrhose nicht als Grund derselben ansehen." Ob nun das Urobilin selbst hepato- oder enterogenen Ursprungs ist, ändert nichts an der praktischen Verwertbarkeit der Urobilinurie als Frühsymptom.

Während heute die Majorität der Untersucher der alimentären Glykosurie für die Diagnose der Lebererkrankungen keine Bedeutung beimißt, wird die alimentäre Lävulosurie als Funktionsprüfung der Leber nicht mehr angefochten. Sowohl für diese als auch für die Galaktosurieprüfung gilt die Tatsache, daß ihr Wert gerade bei beginnender Leberinsuffizienz von Bedeutung ist. Im Stadium stärkerer Pfortaderstauung wirken die gestörten Resorptionsverhältnisse im Darme derart störend, daß der Wert der Probe dadurch sehr leidet. Deswegen sind auch' die Resultate bei unseren Fällen (die Galaktoseprobe wurde bei 6 Patienten angestellt) nicht recht verwertbar. In 2 Fällen wurde der Galaktoseversuch vor und nach der chirurgischen Behandlung des Ascites durchgeführt. In keinem der beiden Fälle konnte auf diesem Wege eine Besserung der Leberfunktion festgestellt werden. Allerdings betraf der erste Fall eine Patientin, bei welcher die schwersten Stauungszustände entwickelt 
waren. Der zweite wurde noch viel zu früh auf alimentäre Galaktosurie nachgeprüft, I 6 Tage post operationem, zu einer Zeit, wo der Ascites noch durch Punktion entleert werden mußte und sich noch keine Kollateralen gebildet hatten. Es wird daher von Interesse sein, künftighin die Nachprüfung auf alimentäre Galaktosurie 3-6 Monate nach der Operation vorzunehmen, um eine etwaige Besserung der Leberfunktion nachzuweisen. Diese Probe ist als die bestausgebaute für diesen $Z$ weck am geeignetsten, denn ihre positiven Resultate sprechen, wie die Erfahrungen gezeigt haben, bereits in einem Stadium für das Bestehen einer Leberzirrhose, wo uns die klinische Untersuchung kaum einen Fingerzeig gibt.

Wenn man auch nicht geneigt sein wollte, auf die eine oder die andere der Funktionsprüfungsmethoden allzu großes Gewicht zu legen, so stellen sie im Verein mit den klinischen Symptomen der initialen Leberzirrhose (das präascitische Ödem, häufige Blutbefunde im Stuhl, initiale Hämatomesis, Milztumor und die sonstigen Allgemeinsymptome) ein Rüstzeug dar, mit welchem man wohl hoffen darf, die Zirrhosen schon früher zu erkennen und sie einer Operation zuzuführen, als deren Resultat ein häufigeres Ausbleiben des Ascites mit größerem Rechte als bisher erwartet werden kann.

\section{Zusammenfassung:}

I. An der II. chirurgischen Klinik wurden von I906-I9I2 I 4 Fälle von Ascites behandelt. Die Erkrankungen, bei welchen die Intervention Erfolg hatte, waren beginnende Leberzirrhosen, tuberkulöse Peritonitiden und ein Fall, wo der Ascites die Folge von Tricuspidalinsuffizienz gewesen ist.

2. Der weitere Verlauf, sowie die Autopsia in vivo und in mortuo bei den anderen Fällen zeigte, daß eine schärfere Dia. gnosen- und Indikationsstellung bessere Operationserfolge erwarten ließe. Insbesondere müßte das Anfangsstadium der Leberzirrhose häufiger diagnostiziert werden.

3. In einem Falle, wo die Ruottesche Operation zur Anwendung kam (es bestand die klinische Diagnose Leberzirrhose), starb die Patientin an Miliartuberkulose. Es hatte bei ihr eine tuberkulöse Peritonitis bestanden. Es wird auf die Gefährlichkeit 
dieser Operation hingewiesen für diejenigen Fälle, wo als Ursache des Ergusses nicht mit Sicherheit 'Tuberkulose des Peritoneums oder Peritonealcarcinose ausgeschlossen werden kann.

4. Mit Rücksicht auf die Wichtigkeit, welche der Diagnose initialer Leberzirrhose zukommt, werden die Anfangssymptome dieser Krankheit kurz zusammengefaßt, sowie die funktionellen Prüfungsmethoden der Leber angeführt.

\section{Literaturverzeichnis.}

I. Koch, C. F, A. (Groningen). 2. Congrès de la soc. internat. de chirurgie. Bruxelles igo8.

2. La denberger. Würzburger Abhandlungen Bd. 8 .

3. Monprofit (Angers). 17. Chir.-KongreB 1904.

4. Bunge. Klin. Jahrb. I905, Bd. I4.

5. Sinclair White. Brit. med. Journ. Igo6, p. I287.

6. Octtinger, „Beitrag z. Talmaschen Op.“ (I.-D. Breslau 1907.)

7. Bircher. Med. Klin. I909, Bd. 5.

8. Greenough. American Journal of medical sciences 1912 .

9. MacD on ald. Med. News 1889, October.

10. Hawkins. Medical Press 1908, April.

II. I. a nti er. Journal de méd. de Bordeaux 1908, Nr. $4^{\circ}$.

12. Cabot. American Journal of med. sciences 1912 .

I3. Thierfelder in Ziemssens Handbuch 1878 .

14. B rist ow e (zit. nach Cheadle, Br. m. J. I892).

15. F a g g e. Prim. and Pract. J. med. 3. edit., p. 328.

16. Pel, P. K. (Amsterdam), „Die Krankheiten der Leber“.

17. E, wald, C. A., „Die Leberkrankheiten“. Lcipzig, Thieme, 1913.

I8. 'T a Ima, S. Zeitschr. f. klin. Med., Bd. 27. - Berl. klin. Wochenschr. 1898, Nr. 38 ; 1900, Nr. 3 r.

19. Drummond and Mor ison. Brit. med. Journ. 1896, Sept. I9.

20. Ruotte. Lyon médical 1907, Nr.40.

21. O $\mathrm{mi}$ und Ito. Deutsche Zeitschr. f. Chir., Bd. 62.

22. O mi. Beitr. z. Chir., Bd. 63 .

23. Delagrenière (Mans). Sem. médicale 1904, Nr. 42.

24. Morosowa. Ref. Zentralbl. f. Chir. IgI3.

25. N a rath. Zentralbl. f. Chir. 1905.

26. Evler. Med. Klinik I9I3, Nr.6.

27. L a mb otte. Semaine médicale 1905, p. I9.

28. Tavel. Zit. nach F. Franke (loc. cit.).

29. Paterson. The Lancet 1910, Okt. 29.

30. Franke, F. Arch. f. klin. Chir., Bd. 93.

3r. Henschen. Zentralbl. f. Chir. 1913, Nr. 2. 
32. Perim off. Zentralbl. f. Chir. I913, Nr. x.

33. Francke (Rostock). 40. Versammlg. d. Deutsch. Ges. f. Chir. Berlin, April IgII.

34. Vida 1. Sem. médicale I907. Französ. Chirurgenkongreß.

35. Villard et Tavernier. Lyon médical I9Io, p. III3.

36. Cha uffard. Traité de Méd. Tome III, p. 82 I.

37. Lieblein. Mitt. a. d. Grenzgeb., Bd. I8, Heft 5.

38. Axisa. Zeitschr. f. innere Med. rgr I, Nr. 2.

39. Hoppe-Seyler in Nothnagels Handbuch 1913.

4o. Litt en. Zeitschr. f. klin. Med., Bd. 5, Heft 1 .

4r. Lange, „Ein Beitrag zur Statistik ..." Zit. nach Nothnagel.

42. M a y o, W. J. Brit. med. Journ. Igo6.

43. Go et j e. D. Zeitschr. f. Chir., Bd. II7, S. 252.

44. Gilbert et Lereboullet. Archives des maladies de l'appareil dig. et de la nutrition igra.

45. Gläs sn er. Zeitschr. f. exp. Path. u. Ther. I907, S. 336.

46. Ebstein (in Ebstein und $\mathrm{Schwalbe}$, Handbuch d. prakt. Med.). 